ACCEPTED MANUSCRIPT

\title{
Towards single electron transistor-based photon detection with microplasma-enabled graphene quantum dots
}

To cite this article before publication: Pei-Chun Yeh et al 2021 Nanotechnology in press https://doi.org/10.1088/1361-6528/ac2845

\section{Manuscript version: Accepted Manuscript}

Accepted Manuscript is "the version of the article accepted for publication including all changes made as a result of the peer review process, and which may also include the addition to the article by IOP Publishing of a header, an article ID, a cover sheet and/or an 'Accepted Manuscript' watermark, but excluding any other editing, typesetting or other changes made by IOP Publishing and/or its licensors"

This Accepted Manuscript is @ 2021 IOP Publishing Ltd.

During the embargo period (the 12 month period from the publication of the Version of Record of this article), the Accepted Manuscript is fully protected by copyright and cannot be reused or reposted elsewhere.

As the Version of Record of this article is going to be / has been published on a subscription basis, this Accepted Manuscript is available for reuse under a CC BY-NC-ND 3.0 licence after the 12 month embargo period.

After the embargo period, everyone is permitted to use copy and redistribute this article for non-commercial purposes only, provided that they adhere to all the terms of the licence https://creativecommons.org/licences/by-nc-nd/3.0

Although reasonable endeavours have been taken to obtain all necessary permissions from third parties to include their copyrighted content within this article, their full citation and copyright line may not be present in this Accepted Manuscript version. Before using any content from this article, please refer to the Version of Record on IOPscience once published for full citation and copyright details, as permissions will likely be required. All third party content is fully copyright protected, unless specifically stated otherwise in the figure caption in the Version of Record.

View the article online for updates and enhancements. 


\section{Towards Single Electron Transistor-Based Photon Detection with Microplasma-Enabled Graphene Quantum Dots}

Pei-Chun Yeh ${ }^{\mathrm{a}}$, Genki Ohkatsu ${ }^{\mathrm{b}}$, Ryo Toyama ${ }^{\mathrm{b}}$, Phan Trong Tue ${ }^{\mathrm{b}}$, Kostya (Ken) Ostrikov, ${ }^{\mathrm{c}}$ Yutaka Majima $^{b^{*}}$, and Wei-Hung Chiang ${ }^{\mathrm{a}}$ *

a Department of Chemical Engineering, National Taiwan University of Science Technology, Taipei 10607, Taiwan

baboratory for Materials and Structures, Institute of Innovative Research, Tokyo Institute of Technology, Yokohama 226-8503, Japan

${ }^{\mathrm{c}}$ School of Chemistry and Physics and QUT Centre for Materials Science, Queensland University of Technology (QUT), Brisbane, QLD, 4000 Australia

\section{ABSTRACT}

Single-electron transistors (SETs) represent a new generation of electronic devices with high charge sensitivity, high switching speed, and low power consumption. Here a simple and controlled fabrication of graphene quantum dot (GQD)-based SETs for photon detectors has been demonstrated. The plasmasynthesized GQDs exhibit stable photoluminescence and are successfully used as the Coulomb islands between heteroepitaxial spherical-gold/platinum $(\mathrm{HS}-\mathrm{Au} / \mathrm{Pt})$ nanogap electrodes. The as-fabricated GQD-SETs enable photon detection with $410 \mathrm{~nm}$ excitation owing to the ability of GQDs to generate photoluminescence emission.

Keywords: Transistor; Graphene Quantum Dots; Plasmas; Synthesis; Photon Detection

\footnotetext{
*Corresponding author emails : whchiang@ mail.ntust.edu.tw ORCID:0000-0002-6350-6696 (W.H.C.); majima@msl.titech.ac.jp ORCID: 0000-0002-5108-1934 (Y. M)
} 
Single-electron transistors (SETs) represent a new class of electronic devices and have been attracted a lot of attention due to their exceptional properties including low power consumption, high charge sensitivity, and multigate logic operation. ${ }^{1,2,3}$ The SETs have been shown promising potential for quantum computing, photon detectors, and nanooptoelectronics. ${ }^{1,2,3}$ In principle, the high charge sensitivity of SETs originates from the change in the offset charge $\left(Q_{0}\right)$ on Coulomb island. When $Q_{0}$ changes from $n$ e to $(n \pm 1 / 2)$ e (e: unit charge, $n$ : integer), the current starts to flow owing to breaking Coulomb blockade condition. Moreover, as the bias voltage between the source and drain is increased, an electron can pass through the Coulomb island. If a photon excites the SETs consisting of Coulomb island and surrounding components, the surrounding components tends be charged, leading the change in $Q_{0}$ on Coulomb island to realize the photon detection by current owing to change in Coulomb blockade condition. Therefore, the materials of the Coulomb islands play a key role on the SET properties for photon detection. Recent studies demonstrate that graphene quantum dots (GQDs), a zero-dimensional (OD) graphene-like nanomaterials with lateral dimensions typically less than $10 \mathrm{~nm}$, possess superior physical and chemical properties including large surface area, high electronic mobility, tunable bandgaps, stable photoluminescence and chemical stability. ${ }^{4,5}$ It has been shown that GQDs can be used as the Coulomb islands within the double-barrier tunneling junction in SETs and present high potential in SET fabrication. ${ }^{6,7}$ In addition, GQDs can act as an active materials for photon detectors with high photocurrents based on their unique optical properties. ${ }^{8}$ The development of fabrication for GQD-based SETs is important for fundamental study and applications.

In this letter, we report a simple fabrication of GQD-based SETs for photon detectors. Colloidal GQDs were initially synthesized from starch in liquid phase at ambient condition using microplasmas and then used as the active materials of Coulomb island to fabricate SETs for photon detector study. Microplasmas are gas discharges confined to electrode geometries less than $1 \mathrm{~mm}$ and can be operated stably at ambient pressure, enabling its use for liquid phase processing at ambient conditions. ${ }^{9,10}$ When microplasmas interact with liquid solutions, energetic species including radicals, ions and electrons formed in the microplasma can initiate non-thermal chemical or electrochemical-related reactions in the solutions $^{11}$, leading colloidal nanomaterials synthesis. ${ }^{12,13}$ Recently, we have established fabrication processes of heteroepitaxial spherical-gold/platinum ( $\mathrm{HS}-\mathrm{Au} / \mathrm{Pt}$ ) nanogap electrodes with $5 \mathrm{~nm}$ in top radii and $0.7 \mathrm{~nm}$ in gap separation by using electron beam lithography (EBL, Elionix, ELS-500$\mathrm{EX})^{14,15}$ and our unique electroless-Au plating (ELGP) ${ }^{16,17}$. Based on HS-Au/Pt nanogap electrodes, we have demonstrated single-molecule single-electron transistor operation. ${ }^{18}$ Here, we introduce a GQD between $\mathrm{HS}-\mathrm{Au} / \mathrm{Pt}$ nanogap electrodes, and demonstrate photon detecting properties of GQD-based 
SETs. The GQD-based SETs show high potential in photon detection based on their exceptional optical properties.

GQD were synthesized in aqueous solutions using a direct current (dc) microplasma electrochemical reactor operated at ambient conditions. ${ }^{13,19-21}$ The synthesis details can be found in the Supporting Information. In brief, $6 \mathrm{~mL}$ of the solution mixed with starch $(0.428 \mathrm{~g})$, deionized water (15 $\mathrm{mL}$ ), and 0.1 M aqueous solution of sodium hydroxide $(2.5 \mathrm{~mL})$ was used as the precursor. A flow of $25 \mathrm{sccm}$ argon (Ar) was introduced in a hollow stainless steel capillary (i.d. $=180 \mu \mathrm{m}$ ) to facilitate the formation of a microplasma with a constant current of $9.6 \mathrm{~mA}$, and the process time was kept at $1 \mathrm{~h}$. After the plasma synthesis, the solution was purified with $10 \mathrm{~mL}$ of ethanol for $10 \mathrm{~min}$. , and then vacuum filtrated with the filter membrane (polyvinylidene difluoride (PVDF) membrane filter; pore size: $0.2 \mu \mathrm{m}$, diameter: $47 \mathrm{~mm}$, Pall cooperation) to remove the unreacted starch. The filtered GQD solution was diluted to a concentration of $4 \mathrm{mg} / \mathrm{mL}$ by adding ethanol and used throughout the work. The morphology of as-synthesized GQDs was analyzed by transmission electron microscopy (TEM) as shown in Fig. 1(a)-(c). TEM was performed using a cold-field emission Cs-corrected TEM (JEOL, ARM-200F) with an accelerating voltage of $200 \mathrm{kV}$. TEM samples were prepared from solution drycasting of the colloidal solution on carbon-coated copper grids (300 mesh, Ted Pella Inc.). As revealed by TEM image shown in Fig. 1(a), the GQDs exhibit spherical particle-like nanostructures with averaged diameter of $3.6 \mathrm{~nm}$ and a narrow size distribution (size histogram is shown as Fig. S1). Highresolution TEM (HRTEM) images further shows the as-synthesized GQDs were highly crystalline (Fig. 1(b)) and with $0.21 \mathrm{~nm}$ graphene lattice (Fig. 1(c)). The photoluminescence (PL) intensity of the synthesized GQDs as a function of excitation and emission was measurement by PL spectroscopy, and the obtained PL map shows one maximum PL intensity (shown in red) with 420 and $505 \mathrm{~nm}$ of excitation and emission (Supporting Information Fig. S2), respectively, which represents a radiative recombination center and presented excitation-dependent properties. The broad range of the radiative recombination center could be attributed to various energy levels induced by surface functional groups, defects and edges states. ${ }^{22}$

To fabricate ultrafine Pt-based nanogap electrodes, electron-beam lithography (EBL) processes were used. Electron-beam (e-beam) resist was coated on a $\mathrm{SiO}_{2} / \mathrm{Si}$ substrate through spin coating. Ultrafine electrode patterns with various nanogap designs and for different dose exposures were obtained by using EBL apparatus. After EBL resist development, titanium (Ti) and Pt were evaporated to form 3 and $10 \mathrm{~nm}$ thick layers, respectively, on the $\mathrm{SiO}_{2} / \mathrm{Si}$ substrate by e-beam deposition. After a resist liftoff process, ultrafine Pt-based nanogap electrodes were obtained. ${ }^{14}$ Then, ELGP process was 
introduced to prepare $\mathrm{HS}-\mathrm{Au} / \mathrm{Pt}$ nanogap electrodes. ${ }^{15-17}$ In brief, for removing the organic contaminants or residual resist on Pt surface, the Pt-based nanogap electrodes were first cleaned by immersing in an acetone/ethanol mixture followed by UV-ozone cleaning. To prepare ELGP solution, Au sheet $(99.99 \%, 65 \mathrm{mg})$ was dissolved in $3 \mathrm{ml}$ of the commonly used medical iodine tincture $\left(\mathrm{I}_{2}\right.$ and $\mathrm{KI}$ in ethanol) with ultrasonic agitation and then, $\mathrm{L}(+)$-ascorbic acid was added as a reducing agent in the Au-dissolved iodine tincture solution until saturation. Finally, ELGP on the Pt surface was conducted by simply immersing cleaned Pt electrodes or Pt-based nanogap electrodes into the ELGP solution, which was diluted 1000 times by DI water $(8 \mathrm{ml}, 18.1 \mathrm{M} \Omega)$. After immersion for the plating, the ELGP Pt electrodes and ELGP Pt-based nanogap electrodes were rinsed in DI water and acetone/ethanol mixture for stopping Au plating. Fabrication of GQD-based SET devices is as follows: After chip cleaning, GQD solution was dropped $5 \mu \mathrm{L}$ each time onto the chip via naturally dry, then repeated 10, 20 and 60 times, respectively. Afterwards, electrical measurements were performed by using a semiconductor parameter analyzer (Agilent, B1500) and a probe station with He-refrigerator (Nagase Techno-Engineering, GRAIL 10-LOGOS01S) in a vacuum $\left(10^{-5} \mathrm{~Pa}\right)$ at $9 \mathrm{~K}^{1-3}$ The SEM image is observed as shown in Fig. 1(d). It presents the clear source, drain, side-gate 1, and side-gate 2 electrodes. In the other hand, the gap separation is less than $5 \mathrm{~nm}$ which is corresponding to the average size of GQD (3.6 nm), suggesting that the gap separation of device is suitable for the GQD particle. First, we put 20 drops of GQD solution to prepare the devices, and to prove the devices based on GQD are with SET characteristics. There are some identifications below.

The drain current-drain voltage $\left(I_{\mathrm{d}}-V_{\mathrm{d}}\right)$ characteristics of a SET with 20 drops of GQD solution with varying a gate voltage $\left(V_{\mathrm{g}}\right)$ simultaneously applied from side gate 1 , side gate 2 and Si back gate from -1.5 to $1.5 \mathrm{~V}$ as shown in Fig. 2(a). The clear Coulomb blockade can be observed, suggesting that GQD particle can act as Coulomb island between drain and source electrodes. It notes that there was no current on the HS-Au/Pt nanogap before the 20 drops. The drain current-gate voltage $\left(I_{\mathrm{d}}-V_{\mathrm{g}}\right)$ characteristics with different drain voltages $\left(V_{\mathrm{d}}\right)$ from -0.1 to $0.1 \mathrm{~V}$ are shown in Fig. 2(b). $I_{\mathrm{d}}$ can be modulated with the varying $V_{\mathrm{g}}$ apparently, regarding that GQD particle works as the effective Coulomb island for current modulation. Note that the parallelogram shape of Coulomb diamonds demonstrates GQD working as a Coulomb island. The Coulomb oscillation is more obvious with increasing $V_{\mathrm{d}}$, which indicated the Coulomb island based on GQD also works as the gate modulation. Based on the stability diagram in Fig. 2(c) and differential stability diagram in Fig. 2(d), they are corresponding to the Coulomb gap in $I_{\mathrm{d}}-V_{\mathrm{d}}$ characteristics (Fig. 2(a)) and $I_{d}-V_{g}$ characteristics (Fig. 2(b)).

After the exploration of SET characterization, we study the relationship between the SET 
characteristics and the density of GQDs. The density increased to 60 drops and decreased to 10 drops, as shown in $I_{\mathrm{d}}-V_{\mathrm{d}}$ characteristics of Fig. 3(a) and 3(c), respectively. Both of the $I_{\mathrm{d}}-V_{\mathrm{d}}$ characteristics show the clear Coulomb blockade. $I_{\mathrm{d}}-V_{\mathrm{g}}$ characteristics of the SETs with 60 and 10 drops are shown in Fig. 3(b) and 3(d), respectively, which also present the Coulomb oscillation in each case. As $I_{\mathrm{d}}-V_{\mathrm{g}}$ characteristics in Figs. 3(b) and 3(d) are not ideal Coulomb oscillation, these SETs should have multiGQDs between the HS-Au/Pt nanogap electrodes. Based on the results, regardless of GQD density, the devices only present the SET characteristics, suggesting that it is a simple procedure to fabricate the SET devices based on GQD. Additionally, the yield of SET devices is from $6 \%$ to $28 \%$ as the GQD density is from 10 drops to 60 drops, respectively. Note that increasing the GQD density can improve the yield efficiently.

Afterwards, the photon detector based on GQD-based SET devices is explored. Based on our PL result (Figure S2), GQDs present the maximum emission intensity under excitation $420 \mathrm{~nm}$, thus, the LED (Lumileds) source of $410 \mathrm{~nm}$ is utilized. In addition, we utilized lock-in amplifier (NF, 5640) to obtain the change in current under intermittent photo irradiation at $520 \mathrm{~Hz}$. We study on relationship between $I_{\mathrm{d}}$ and different parameters include $I_{\mathrm{LED}}$ and $V_{\mathrm{d}}$. First, $I_{\mathrm{LED}}$ is adjusted from 2.8 to $352 \mathrm{~mA}$, and the $\Delta I_{\mathrm{d}}\left(=I_{\mathrm{d}}(\right.$ with LED source $)-I_{\mathrm{d}}($ without LED source $\left.)\right)-$ time dependence were measured under the application of $V_{\mathrm{d}}=-2 \mathrm{~V}$ as shown in Fig. 4(a). The $I_{\mathrm{LED}}$-dependent $\Delta I_{\mathrm{d}}$ property under LED source is shown in Fig. 4(b). $\Delta I_{\mathrm{d}}$ increased with increasing $I_{\mathrm{LED}}$, indicating that the higher photon flux density enhance the photo current. It is also suggested that once GQD particles detect stronger photon density, it would enhance the current directly. It notes that periodic noise with interval of $1 \mathrm{~s}$ in Fig. 4(a) comes from pumping of the He-refrigerator. The $V_{\mathrm{d}}$-dependent $\Delta I_{\mathrm{d}}$ property under LED source is also shown in Fig. 4(c), and the clear linear relationship can be observed, suggesting that GQD-based SET devices show high potential and promising prospect in photon detection.

In summary, we have demonstrated that microplasma-synthesized GQDs can be utilized as Coulomb island in SETs, which present clear SET characteristics. In addition, the devices would only present the multi-GQDs SET characteristics regardless of GQD density, note that it is a simple procedure to fabricate the SET devices using GQD. Furthermore, the increasing density of GQDs can improve the yield efficiently. On the other hand, the photon detector based on GQD-based SET devices is also explored. We find superior sensitivity and linear relationship between $\Delta I_{d}$ and $I_{\mathrm{LED}}$, and between $\Delta I_{d}$ and $V_{\mathrm{d}}$, respectively. The GQD-based SET devices show high potential and promising prospect in photon detection. Our work provides an effective method for SET fabrication and open a new avenue 
for SET fundamental study and applications.

\section{ACKNOWLEDGMENT}

Y. P. C. thanks Tokyo Institute of Technology for the support of Asia-Oceania Strategic Universities (AOSU) Exchange Program. W. H. C. thanks the Ministry of Science and Technology of Taiwan (MOST Grant no. MOST 107-2628-E-011-002-MY3) and National Taiwan University of Science and Technology for the support. Y. M. thanks MEXT Element Strategy Initiative to Form Core Research Center (Grant Number JPMXP0112101001) and BK Plus Program, the Basic Science Research Program (NRF-2014R1A6A1030419).

\section{Author Contributions}

All authors have given approval to the final version of the manuscript. 


\section{Figure captions}

Fig. 1. (a) Low-magnified TEM and (b) HRTEM images of as-synthesized GQDs using microplasmas. (c) HRTEM image of one GQD with $0.21 \mathrm{~nm}$ graphene lattice. (d) SEM image of GQD-based SET device.

Fig. 2. (a) $I_{\mathrm{d}}-V_{\mathrm{d}}$ characteristics of a fabricated SET with 20 drops of GQD solution under different gate voltages, the solid and dashed lines are consecutive forward and backward drain voltage sweeps, respectively. (b) $I_{\mathrm{d}}-V_{\mathrm{g}}$ characteristics of the SET with 20 drops of GQD solution under applying different drain voltage. the solid dashed lines are consecutive forward and backward tri-gate voltage sweeps, respectively. (c) Stability diagram. (d) Differential current stability diagram.

Fig. 3 (a) $I_{\mathrm{d}}-V_{\mathrm{d}}$ characteristics of a fabricated SET with 10 drops of GQD solution, the solid and dashed lines are consecutive forward and backward drain voltage sweeps, respectively. (b) $I_{\mathrm{d}}-V_{\mathrm{g}}$ characteristics of the SET with 10 drops of GQD solution. the solid dashed lines are consecutive forward and backward tri-gate voltage sweeps, respectively. (c) $I_{d}-V_{d}$ characteristics of a fabricated SET with 60 drops of GQD solution. (d) $I_{\mathrm{d}}-V_{\mathrm{g}}$ characteristics of the SET with 60 drops of GQD solution

Fig. 4. (a) $\Delta I_{d}$ - time characteristics of a fabricated SET with 20 drops of GQD solution under different

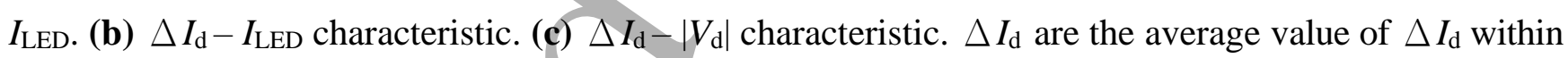
$17 \mathrm{~s}$. 


\section{References}

1 K. Maeda, N. Okabayashi, S. Kano, S. Takeshita, D. Tanaka, M. Sakamoto, T. Teranishi, and Y. Majima, ACS Nano 6, 2798 (2012).

2 N. Okabayashi, K. Maeda, T. Muraki, D. Tanaka, M. Sakamoto, T. Teranishi, and Y. Majima, Appl. Phys. Lett. 100, 033101 (2012).

3 Y. Majima, G. Hackenberger, Y. Azuma, S. Kano, K. Matsuzaki, T. Susaki, M. Sakamoto, and T. Teranishi, Sci. Technol. Adv. Mater. 18, 374 (2017).

4 Xuewan Wang, Gengzhi Sun, Nan Li, and Peng Chen, Chem. Soc. Rev. 45, 2239 (2016).

5 Xin Ting Zheng, Arundithi Ananthanarayanan, Kathy Qian Luo, and Peng Chen, Small 11, 1620 (2015).

6 L. J. Wang, G. Cao, T. Tu, H. O. Li, C. Zhou, X. J. Hao, Z. Su, G. C. Guo, H. W. Jiang, and G. P. Guo, Appl. Phys. Lett. 97, (2010).

7 Stefan Fringes, Christian Volk, Caroline Norda, Bernat Terrés, Jan Dauber, Stephan Engels, Stefan Trellenkamp, and Christoph Stampfer, Phys. Status Solidi B 248, 2684 (2011).

8 Hiroyuki Tetsuka, Akihiro Nagoya, Takanori Fukusumi, and Takayuki Matsui, Adv. Mater. 28, 4632 (2016).

9 W. H. Chiang, D. Mariotti, R. M. Sankaran, J. G. Eden, and K. Ostrikov, Adv. Mater. 32, 1905508 (2020)

10 Davide Mariotti and R. Mohan Sankaran, J. Phys. D Appl. Phys. 43, 323001 (2010).

11 Wei-Hung Chiang, Carolyn Richmonds, and R. Mohan Sankaran, Plasma Source Sci. Technol. 19, $034011(2010)$.

12 C. Richmonds and R. M. Sankaran, Appl. Phys. Lett. 93, 131501 (2008).

13 J. S. Yang, D. Z. Pai, and W. H. Chiang, Carbon 153, 315 (2019).

14 Y. Y. Choi, T. Teranishi, and Y. Majima, Appl. Phys. Express 12, 025002 (2019).

15 Y. Y. Choi, A. Kwon, and Y. Majima, Appl. Phys. Express 12, 125003 (2019).

16 Y. Yasutake, K. Kono, M. Kanehara, T. Teranishi, M. R. Buitelaar, C. G. Smith, and Y. Majima, Appl. Phys. Lett. 91, 203107 (2007).

17 V. M. Serdio, Y. Azuma, S. Takeshita, T. Muraki, T. Teranishi, and Y. Majima, Nanoscale 4, 7161 (2012).

18 S. J. Lee, J. Kim, T. Tsuda, R. Takano, R. Shintani, K. Nozaki, and Y. Majima, Appl. Phys. Express 12, 125007 (2019).

19 Y. C. Chen, W. H. Chiang, D. Kurniawan, P. C. Yeh, K. Otake, and C. W. Kung, ACS Appl. Mater. Interfaces 11, 35319 (2019). 
20 J. S. Yang, Y. C. Chang, Q. H. Huang, Y. Y. Lai, and W. H. Chiang, Chem. Comm. 56, 10365 (2020).

21 P. C. Yeh, S.H. Yoon, D. Kurniawan, Y.G. Chung, and W. H. Chiang, ACS Appl. Nano Mater. 4, 5636 (2021).

22 S. Hu, A. Trinchi, P. Atkin, and I. Cole, Angew. Chem. Int. Ed. Engl. 54, 2970 (2015). 


\section{Figures}
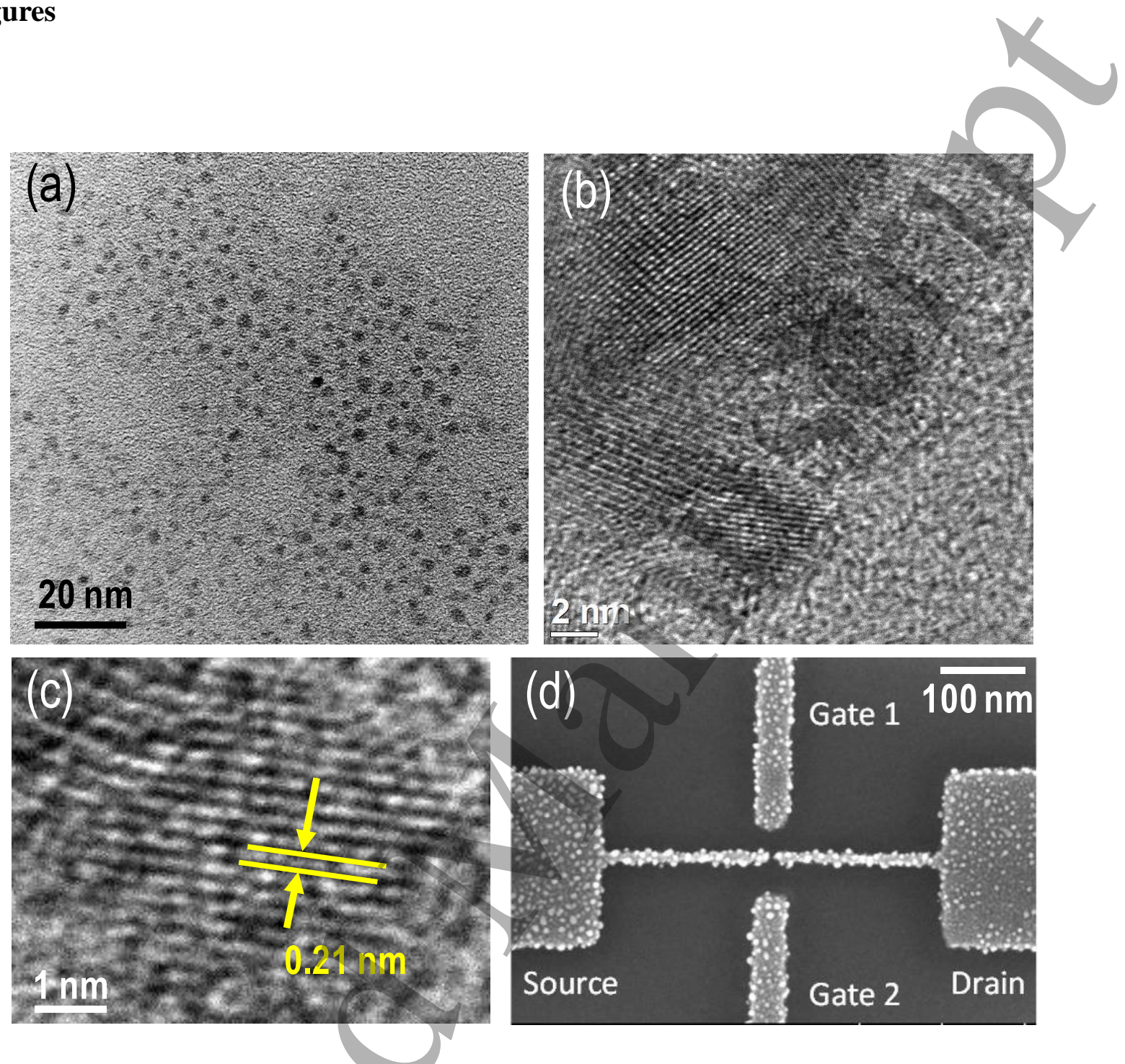

Figure 1. (a) Low-magnified TEM and (b) HRTEM images of as-synthesized GQDs using microplasmas. (c) HRTEM image of one GQD with $0.21 \mathrm{~nm}$ graphene lattice. (d) SEM image of GQDbased SET device.

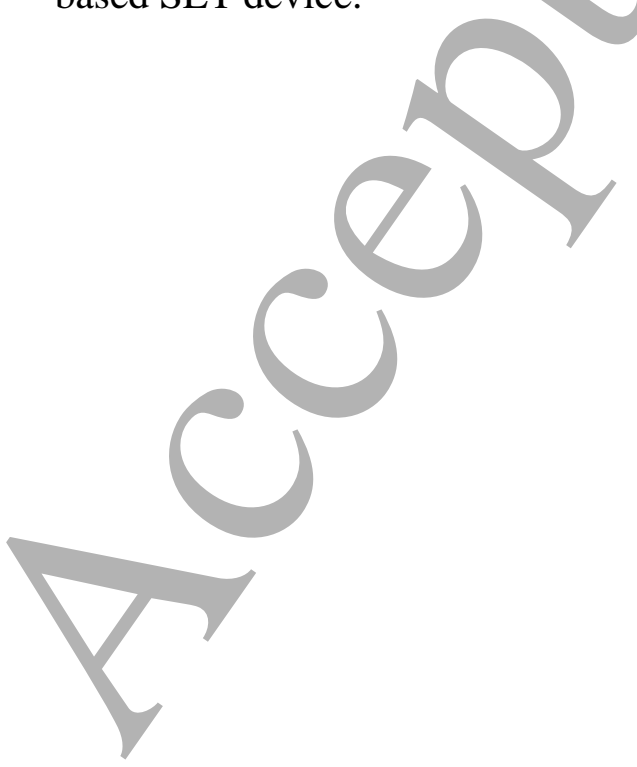




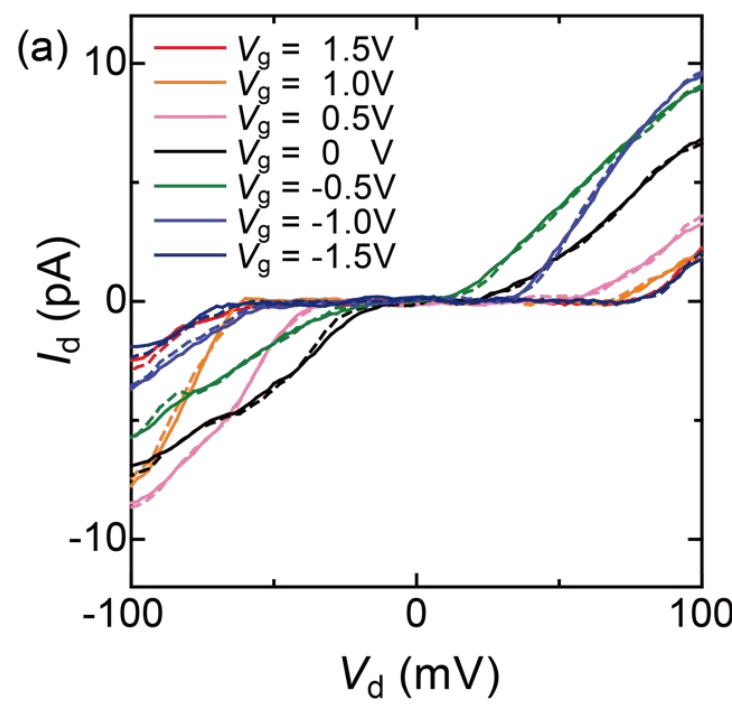

(b)
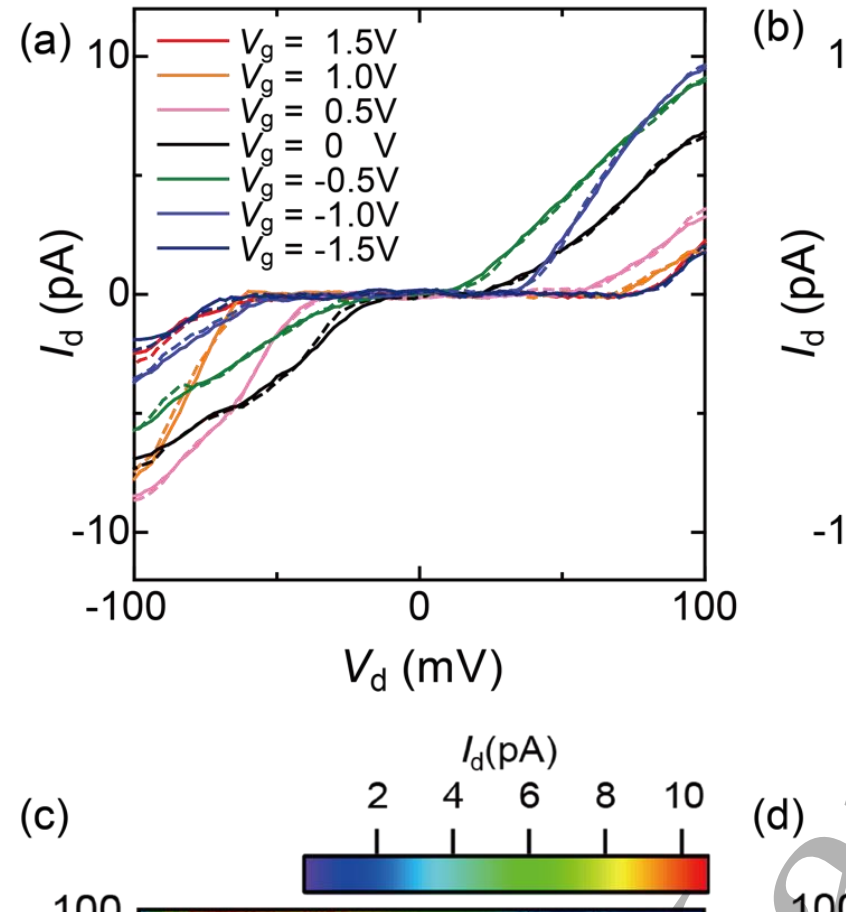

(d)
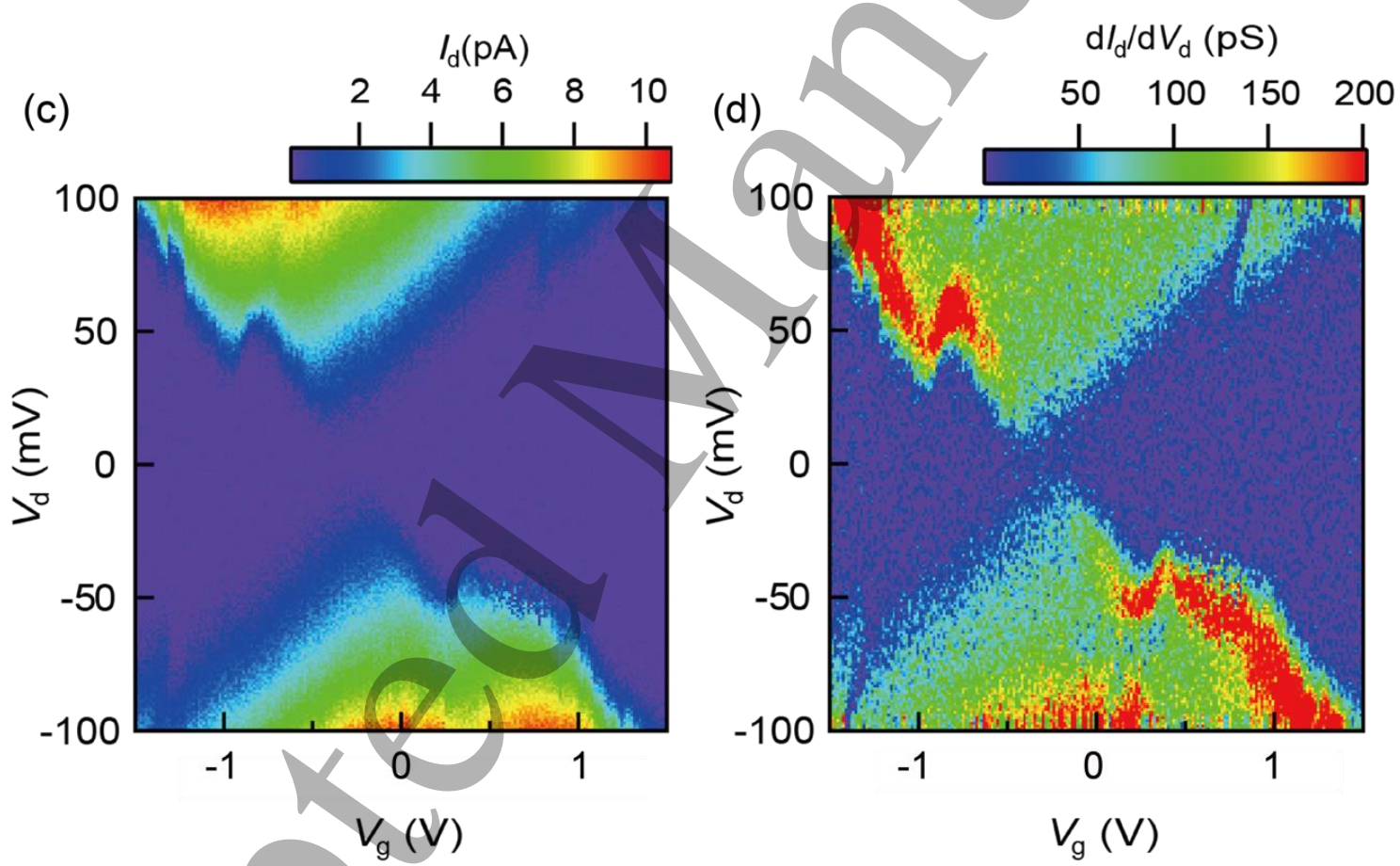

Figure 2. (a) $I_{\mathrm{d}}-V_{\mathrm{d}}$ characteristics of a fabricated SET with 20 drops of GQD solution under different gate voltages, the solid and dashed lines are consecutive forward and backward drain voltage sweeps, respectively. (b) $I_{\mathrm{d}}-V_{\mathrm{g}}$ characteristics of the SET with 20 drops of GQD solution under applying different drain voltage. the solid dashed lines are consecutive forward and backward tri-gate voltage sweeps, respectively. (c) Stability diagram. (d) Differential current stability diagram. 

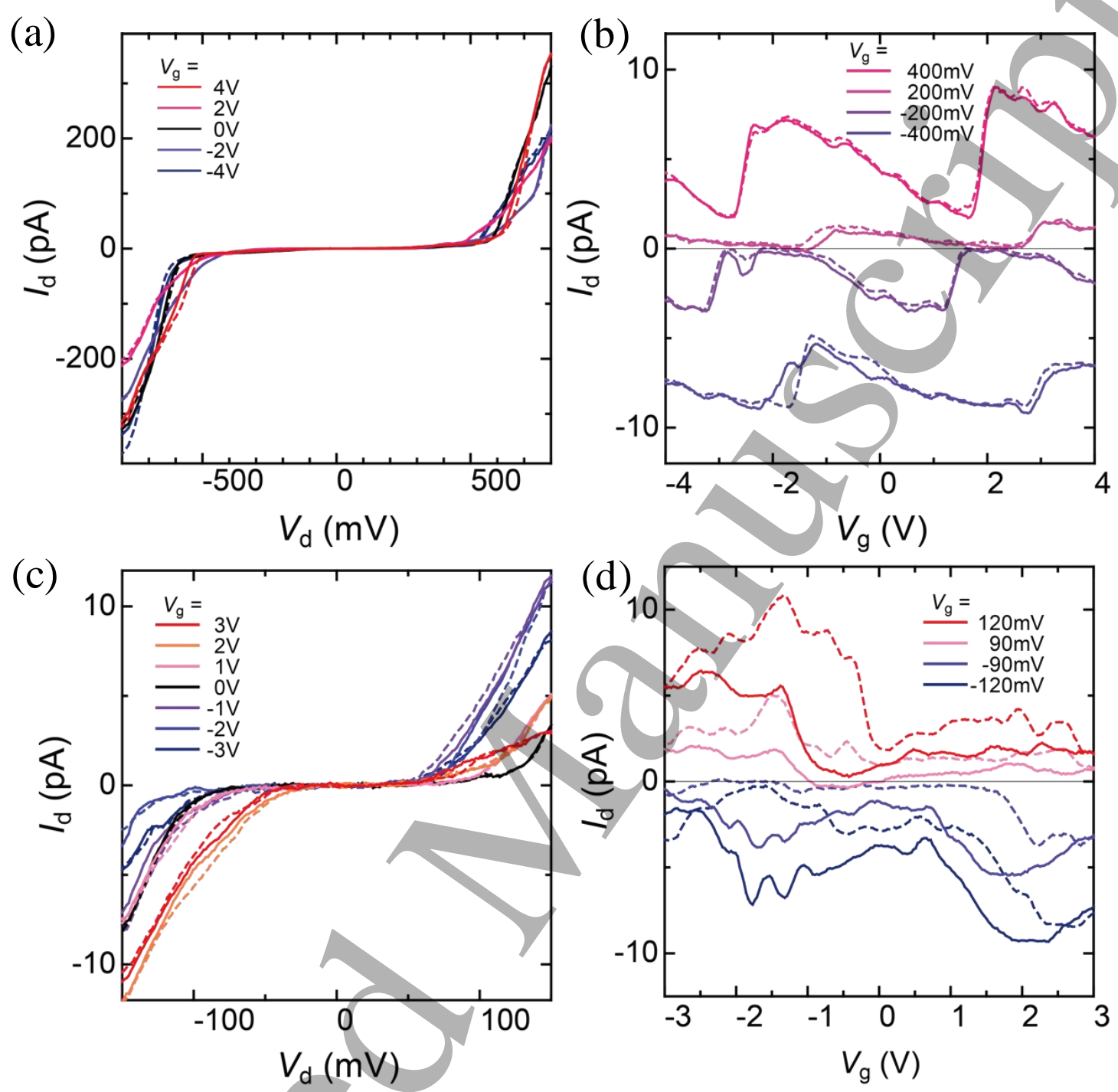

Figure 3 (a) $I_{\mathrm{d}}-V_{\mathrm{d}}$ characteristics of a fabricated SET with 10 drops of GQD solution, the solid and dashed lines are consecutive forward and backward drain voltage sweeps, respectively. (b) $I_{\mathrm{d}^{-}} V_{\mathrm{g}}$ characteristics of the SET with 10 drops of GQD solution. the solid dashed lines are consecutive forward and backward tri-gate voltage sweeps, respectively. (c) $I_{\mathrm{d}}-V_{\mathrm{d}}$ characteristics of a fabricated SET with 60 drops of GQD solution. (d) $I_{\mathrm{d}}-V_{\mathrm{g}}$ characteristics of the SET with 60 drops of GQD solution. 
(a)

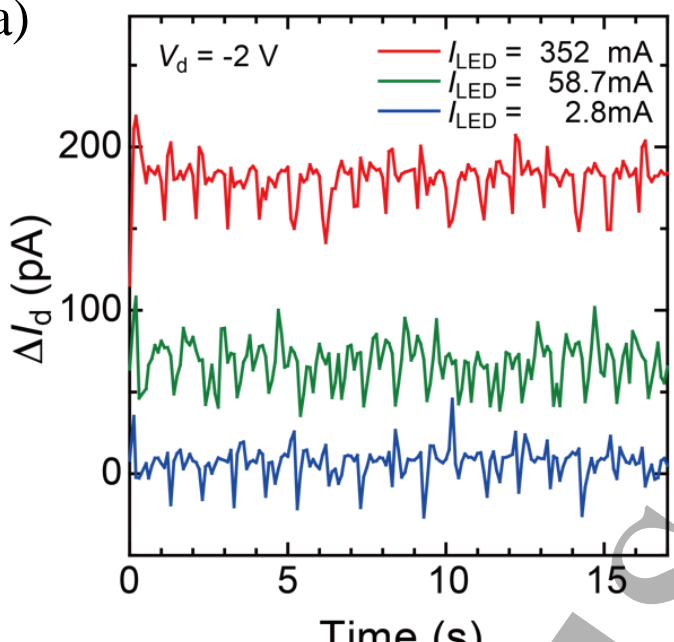

(b)

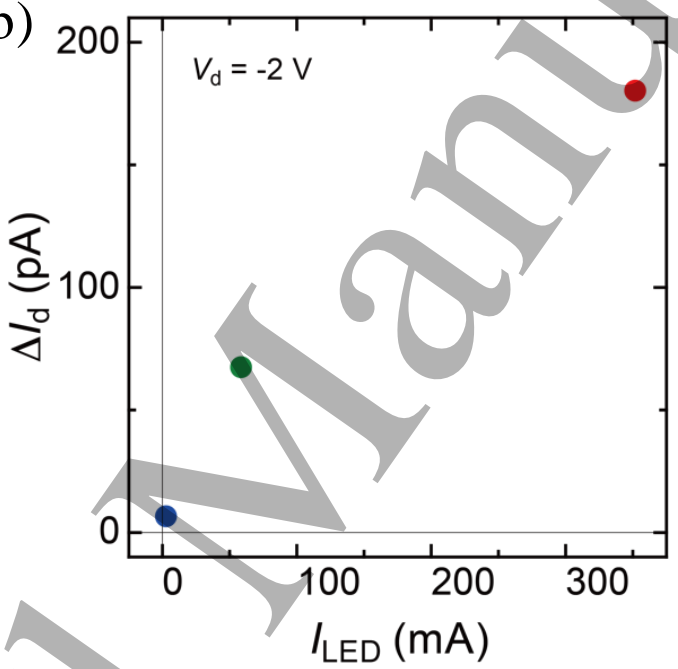

(c)

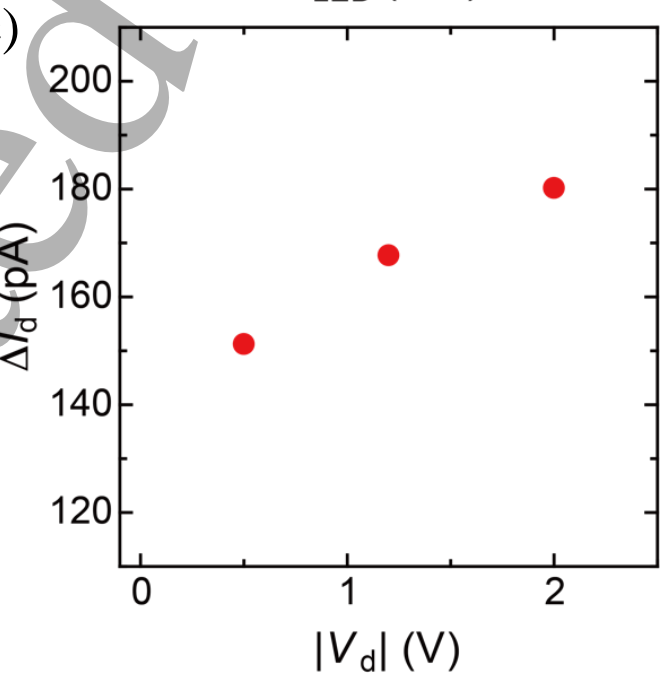

Figure 4. (a) $\Delta I_{d}$ - time characteristics of a fabricated SET with 20 drops of GQD solution under different $I_{\mathrm{LED}}$. (b) $\Delta I_{\mathrm{d}}-I_{\mathrm{LED}}$ characteristic. (c) $\Delta I_{\mathrm{d}}-\left|V_{\mathrm{d}}\right|$ characteristic. $\Delta I_{\mathrm{d}}$ are the average value of $\Delta I_{\mathrm{d}}$ within $17 \mathrm{~s}$. 